\title{
The Drosophila SH2-SH3 adapter protein Dock is expressed in embryonic axons and facilitates synapse formation by the RP3 motoneuron
}

\author{
Chand J. Desai ${ }^{1,2, *}$, Paul A. Garrity ${ }^{3}$, Haig Keshishian ${ }^{5}$, S. Lawrence Zipursky ${ }^{3,4}$ and Kai Zinn",* \\ ${ }^{1}$ Division of Biology, California Institute of Technology, Pasadena, CA 91125, USA \\ 2Department of Pharmacology, Vanderbilt University Medical Center, Nashville TN 37232, USA \\ ${ }^{3}$ Department of Biological Chemistry, UCLA School of Medicine, Los Angeles, CA 90095, USA \\ ${ }^{4}$ Howard Hughes Medical Institute, UCLA School of Medicine, Los Angeles, CA 90095, USA \\ ${ }^{5}$ Biology Department, Yale University, New Haven, CT 06511, USA \\ *Authors for correspondence (e-mail: zinnk@cco.caltech.edu; chand.desai@mcmail.vanderbilt.edu)
}

Accepted 13 January; published on WWW 3 March 1999

\section{SUMMARY}

The Dock SH2-SH3 domain adapter protein, a homolog of the mammalian Nck oncoprotein, is required for axon guidance and target recognition by photoreceptor axons in Drosophila larvae. Here we show that Dock is widely expressed in neurons and at muscle attachment sites in the embryo, and that this expression pattern has both maternal and zygotic components. In motoneurons, Dock is concentrated in growth cones. Loss of zygotic dock function causes a selective delay in synapse formation by the RP3 motoneuron at the cleft between muscles 7 and 6 . These muscles often completely lack innervation in late stage $\mathbf{1 6}$ dock mutant embryos. RP3 does form a synapse later in development, however, because muscles 7 and 6 are normally innervated in third-instar mutant larvae. The absence of zygotically expressed Dock also results in subtle defects in a longitudinal axon pathway in the embryonic central nervous system. Concomitant loss of both maternally and zygotically derived Dock dramatically enhances these central nervous system defects, but does not increase the delay in RP3 synaptogenesis. These results indicate that Dock facilitates synapse formation by the RP3 motoneuron and is also required for guidance of some interneuronal axons The involvement of Dock in the conversion of the RP3 growth cone into a presynaptic terminal may reflect a role for Dock-mediated signaling in remodeling of the growth cone's cytoskeleton.

Key words: Adapter protein, Dock, Nck, Neuromuscular junction, Growth cone, Synaptogenesis, Tyrosine phosphorylation, Drosophila

\section{INTRODUCTION}

Neuronal growth cones are directed along pathways towards their synaptic targets by guidance cues that are expressed on the cell surfaces over which they travel or secreted from nearby cells. In the Drosophila embryo, each identified motor neuron extend its axon along a stereotypical route and always innervates the same muscle fiber (for review see Keshishian et al., 1996). After motor growth cones reach the sites of future neuromuscular junctions, they continue to explore the surfaces of the muscle fibers for several hours and there are no striking changes in growth cone morphology (Yoshinara et al., 1997). Neurotransmitter is released during this exploratory period, however, and glutamate receptors on the muscle surface begin to cluster at the future synaptic zones about an hour after growth cone contact (Broadie and Bate, 1993). Thus, the growth cone already has attributes of a functional presynaptic terminal prior to any morphological manifestation of synaptic maturation.

During late embryogenesis, motor growth cones begin to form rudimentary synapses known as pre-varicosities and these later constrict into smaller swollen regions that appear to be individual varicosities (Yoshinara et al., 1997). The motor nerve terminals are fully functional by hatching. The smooth transition between growth cone exploration and synaptic maturation in this system suggests the possibility that the same kinds of signaling events could be involved in axon guidance and in synapse formation.

Signaling via control of tyrosine phosphorylation is an important mechanism in axon guidance in both Drosophila and vertebrate systems. The Drosophila Derailed receptor tyrosine kinase (RTK) is required for correct guidance of a subset of interneuronal axons (Callahan et al., 1995). In vertebrates, the graded expression of Eph RTKs on retinal axons and Eph ligands on their tectal synaptic targets supports an instructive role for these molecules in the establishment of topographic maps and genetic studies indicate that Eph kinases are required for guidance of commissural axons in the mouse brain (Holland et al., 1996; Orioli et al., 1996; Park et al., 1997). Receptor tyrosine phosphatases (RPTPs) are also expressed on axons and control many aspects of motor axon guidance and synaptogenesis in Drosophila (Desai et al., 1996, 1997; Krueger et al., 1996).

SH2 and SH3 domain adapter proteins can link tyrosine kinases and phosphatases to cytoskeletal alterations because 
they recruit a variety of proteins into complexes with activated kinases and their phosphorylated substrates. Indeed, a role in linking phosphotyrosine signaling to control of axon guidance has been proposed for the Dock adapter protein, which is localized to photoreceptor $(\mathrm{R})$ growth cones and is essential for $\mathrm{R}$ axon outgrowth in Drosophila larvae (Garrity et al., 1996). Dock comprises three N-terminal SH3 domains and a single C-terminal SH2 domain, and is homologous to the mammalian adapter protein Nck. In this paper, we examine the expression and function of Dock during axonogenesis in the embryo. We find that Dock is expressed in motor axon growth cones and that the absence of zygotic Dock delays formation of a single synapse in the embryonic neuromuscular system.

\section{MATERIALS AND METHODS}

\section{Genetics}

The mutant lines used in this study were previously described (Garrity et al., 1996; Kopczynski et al., 1996). dock and dock lbm mutants for embryo and larval collections were maintained using the $\mathrm{CyO}-\mathrm{lacZ}$ and Gla Bc Elp balancer, respectively. Embryos lacking maternal dock expression were generated using the FRT/FLP system (Chou and Perrimon, 1996).

\section{Immunohistochemistry}

Embryo collections and immunohistochemistry were performed as described in Desai et al. (1996). mAb ID4 (diluted 1:10) was used to visualize motor axons (Van Vactor et al., 1993) in whole-mount embryos, whereas mAb 7G10 (diluted 1:10) was used to detect Fasciclin III (Patel et al., 1987) in live-dissected embryos. Affinitypurified rabbit anti-Dock antiserum (diluted 1:1000) was used to detect Dock (Clemens et al., 1996) in both live-dissected and fixed whole-mount embryos. DSyt2 rabbit anti-synaptotagmin (Syt) antiserum (diluted 1:1000) was used to detect Syt in live-dissected embryos. Embryos homozygous for dock and for dock lbm mutations were identified as lacking the $C y O$ lac $Z$ balancer by using anti- $\beta$ galactosidase ( $\beta$-gal) mAb (Promega, diluted 1:1000), or as those lacking strong embryonic Dock expression. Embryos lacking maternal Dock were identified by their failure to stain with either anti$\beta$-gal mAb or anti-Dock antiserum. Non-staining embryos were restained with mAb 1D4. Third-instar larvae, identified as dock or dock $\mathrm{lbm}$ homozygotes by the absence of the dominant Black Cell phenotype conferred by the Gla Bc Elp balancer, were dissected, fixed and stained using anti-HRP sera as described by Chiba et al. (1993). Whole-mount embryos were filleted before mounting and all specimens were analyzed with a Zeiss Axioplan or an Olympus AX70 microscope using DIC optics. Figures were created by scanning negatives and creating Photoshop 3.0 files, some of which were merged to generate montages of different focal planes.

\section{Microinjection}

Embryos were collected from $\operatorname{dock}^{P 2} / G l a$ Bc Elp parents on apple juice agar plates, allowed to develop for 16 hours and dechorionated by rolling on double-stick tape. Embryos lacking black cells were dissected and cells filled with Lucifer Yellow dye as described by Chiba et al. (1993).

\section{RESULTS}

\section{Dock is expressed in embryonic axons and growth cones}

Dock protein is expressed in most or all central nervous system
(CNS) axons and cell bodies (Fig. 1A). It can be visualized in motor axons, which exit the CNS via two nerve roots and then branch into five nerves that innervate the body wall muscles (Fig. 2A,D). All five motor nerves are labeled with anti-Dock antisera. The entire length of the intersegmental nerve (ISN) can be visualized using anti-Dock, but expression levels are highest in growth cones. The $\mathrm{SNb}$ (also known as ISNb) nerve is also stained with anti-Dock (Fig. 2D), with highest expression in growth cones (arrowheads), including the RP3 growth cone (red arrowhead), which forms a synapse along the cleft between ventrolateral muscle fibers 7 (also known as VL4) and 6 (VL3).

The darkest anti-Dock staining in the embryo is in body wall muscles where they attach to the epidermis (Fig. 2B-D). The muscle attachment sites appear as lines (long arrows), marking the insertion points of longitudinal muscles such as the dorsal acute (2), lateral longitudinal (4) and ventrolateral muscles (7, 6, 13 and 12; muscles 13 and 12 are also known as VL2 and VL1, respectively), or as spots (small arrows), marking the insertion point of transverse muscle fibers such as the dorsal (18) and lateral transverse muscles (21, 22, 23 and 24). All of the body wall muscle attachment sites appear to express Dock, but those belonging to longitudinally oriented fibers are particularly prominent.

Most sensory neurons in the peripheral nervous system (PNS) express Dock (Fig. 2C), including chordotonal organ neurons (c), multiple dendrite neurons (m), and external sensory and dendritic arborization neurons in the dorsal cluster (d). PNS axons also express Dock, as indicated by the arrowheads in Fig. 2A.

\section{RP3 synapses are often absent in dock mutant embryos}

The observed pattern of Dock expression suggests that Dock may be important for the establishment of neuronal connections and the attachment of muscles to the epidermis. Three mutations that eliminate or reduce Dock expression have been isolated and previously described (Garrity et al., 1996). All three disrupt $\mathrm{R}$ axon guidance and targeting and result in pupal lethality. We now wished to determine the effect of these mutations on the development of the embryonic nervous system.

In contrast to the severe defects in optic lobe innervation observed in dock mutant larvae, embryos that lack zygotic Dock have very subtle nervous system defects. In the ventral nerve cord, the anti-Fasciclin II (FasII) monoclonal antibody (mAb) 1D4 (Van Vactor et al., 1993) stains three pairs of longitudinal axon bundles (Fig. 1D). These bundles are always present in dock embryos, but they appear somewhat thicker and wavier than in wild-type embryos (Fig. 1E), and the outermost bundle is occasionally discontinuous (arrowheads). The thickened, wavy appearance of the longitudinal axon bundles could be caused by intermittent defasciculation. There are also low-penetrance defects in muscle organization $(<10 \%$ of mutant hemisegments have one or more muscle fibers that are missing or abnormally cross over one another), which may be caused by loss of Dock at muscle attachment sites. The penetrance of these muscle phenotypes is not increased in embryos lacking both maternal and zygotic Dock (data not shown). Dock is thus not necessary for attachment of muscle fibers. 
The most-specific defect observed in dock embryos is the variable absence of a single synapse in the neuromuscular system: that formed by the RP3 neuron along the cleft between muscles 7 and 6. In wild-type embryos (Fig. 3A), axons from the RP1, RP3, RP4 and RP5 neurons extend together within the $\mathrm{SNb}$ nerve until they reach the $7 / 6$ cleft. At this point, the RP3 growth cone defasciculates from the other RP axons and makes a sharp medial turn to grow between muscle fibers 7 and 6. Concomitantly, the RP1 and RP4 growth cones form a large presynaptic structure along the nearby edge of muscle 13 (right-pointing black arrowhead in Fig. 3A). Upon reaching the internal surface of muscles 7 and 6, the RP3 growth cone again reorients to extend posteriorly along the cleft, resulting in a long branch perpendicular to the $\mathrm{SNb}$ nerve, as indicated by the red arrowheads in Fig. 3A. Meanwhile, the RP5 axon extends distally across the breadth of muscle fiber 13 and forms synapses at the cleft between muscles 13 and 12. Although the growth cones of RP1 and RP4 arrive at their target first, RP3 usually forms a mature synapse first, while RP5 is usually last. In the late-stage 16 embryo shown in Fig. 3A, the RP5 growth cone is exploring muscle fiber 13 (left-pointing black arrowheads) and beginning to contact the 13/12 cleft.

Mutants homozygous for three different dock alleles as well as those trans-heterozygous for two different combinations of dock alleles all variably lack the RP3 synapse at the 7/6 cleft. Pan-neuronal expression of dock largely restores this innervation (elav-dock; $\operatorname{dock}^{P 1}$, see Table 1). The images in Fig. 3B and $\mathrm{C}$ show the same $\operatorname{dock}^{P 2} /$ dock $^{3}$ mutant embryo in two different focal planes. The three hemisegments in Fig. 3B all lack RP3 synapses (normally located at positions indicated by red arrowheads), which would be visualized in this focal plane (compare to Fig. 3A). Other $\mathrm{SNb}$ growth cones have contacted the 13/12 cleft and are beginning to form synapses there in all three hemisegments (left-pointing black arrowheads), indicating that this embryo is slightly older than the wild-type embryo in Fig. 3A. Note the synapses forming along the proximal edge of muscle 13 beneath muscles 7 and 6 , visible in the deeper focal plane shown in Fig. 3C (arrowheads). These images indicate that, although the RP3 synapse is not present, the other synapses made by $\mathrm{SNb}$ motoneurons are apparently forming in a normal manner in this dock mutant.

When scored relative to wild type using a qualitative scale for synaptic length (see Table 1 legend), the penetrance of the loss of $7 / 6$ cleft innervation is $62 \%$ in dock $^{P 1}$ embryos and $68 \%$ in $\operatorname{dock}^{P 1} /$ dock $^{P 2}$ late stage 16/early stage 17 embryos. In these genotypes, $45 \%\left(\right.$ dock $\left.^{P 1}\right)$ or $62 \%\left(\right.$ dock $^{P 1} /$ dock $\left.^{P 2}\right)$ of $7 / 6$ clefts have no visible synapses at all at this stage, while only $10 \%$ lack visible synapses in wild type. Genotypes involving only the $\operatorname{dock}^{P 2}$ and dock $^{3}$ alleles exhibit lower penetrances of the RP3 synaptogenesis phenotype (Table 1). The differences between the mutant and control (wild-type) values are highly significant, with $P<0.0001$ (Chi-square test) for all genotypes except $\operatorname{dock}^{P 2} /$ dock $^{3}$, for which $P<0.005$.

The $\operatorname{dock}^{P 1}$ and $\operatorname{dock}^{P 2}$ mutations result from independent P-element insertions into the first intron of the dock gene. The difference in the severity of the defects caused by the various alleles may indicate that $\operatorname{dock}^{P 2}$ is not a null allele. The data also show that this defect is specific to the RP3 synapse and does not reflect a global delay in $\mathrm{SNb}$ synapse formation, because the length of the $7 / 6$ cleft synapse relative to the total length of all the synapses made by RP neurons on the ventrolateral muscles is also greatly reduced in dock mutants (Table 1).

\section{Effects of dock mutations on expression of a presynaptic terminal protein at the $7 / 6 \mathrm{cleft}$}

The analysis described above shows that FasII staining is absent or reduced at the 7/6 cleft in many dock mutant hemisegments. In order to prove that this actually reflects the absence of a nascent synapse at the cleft and not downregulation of FasII expression at this site, we stained wild-type and dock mutant embryos with an antiserum against the presynaptic terminal protein synaptotagmin (Syt), which is involved in neurotransmitter release (Perin et al., 1991). At this stage of embryonic development, Syt is expressed in the motor axons as well as at synaptic regions (Fig. 4).

The $7 / 6$ cleft synapse is absent or smaller in stage $16 / 17$ dock $^{P 1}$ mutant hemisegments than in wild type when examined using anti-Syt (Fig. 4). The increased frequency of missing RP3 synapses $\left(37 \%\right.$ for $\operatorname{dock}^{P 1}$ versus $7 \%$ for $\left.\operatorname{dock}^{P l} /+\right)$ and reduced frequency of normally sized synapses (26\% for $\operatorname{dock}^{P 1}$ versus $64 \%$ for $\operatorname{dock}^{P 1} /+$ ) are both highly statistically significant $(P<0.0001$ by Chi-square test; Table 2$)$. Other $\mathrm{SNb}$ synapses appear to form in a normal manner when examined with this marker. Thus, analysis with anti-Syt confirms the conclusions reached from staining with FasII.

\section{Absence of RP3 synapses in dock embryos is due to a delay in synapse formation rather than to errors in axon guidance}

The absence of a synapse between muscles 7 and 6 could be due to impaired RP3 growth cone maturation into a presynaptic terminal, to aberrant RP3 axon guidance or to a more general defect in RP3 differentiation. In order to distinguish between these possibilities, we first examined the expression of the surface protein Fasciclin III (FasIII). FasIII is expressed on two clusters of RP neurons, including RP3, in the CNS of each segment in wild-type embryos (Patel et al., 1987; Chiba et al., 1995; Kose et al., 1997). These clusters are normally positioned and contain the expected number of cell bodies in dock mutant embryos (large arrowheads in Fig. 5A; this is actually a dock lbm double mutant (see below), but dock single mutants look identical). The RP axons also express FasIII, and we observed no misrouted axons (small black arrowheads), suggesting that RP3 differentiates normally and extends an axon along a relatively normal trajectory in dock mutants. Only one RP3 has begun to form a synapse along the 7/6 cleft (horizontal red arrowhead). The $7 / 6$ clefts in the other two hemisegments remain uninnervated (upward red arrowhead). Fig. 5B, which shows a focal plane beneath the $7 / 6$ cleft in the same embryo, reveals that FasIII-expressing growth cones (most likely from RP1 and/or RP4) are already exploring muscle 13 distal to the $7 / 6$ cleft, as indicated by the diagonal arrowheads. The accumulation of FasIII-staining material directly under the $7 / 6$ cleft, indicated by the vertical arrowheads, may represent paused RP3 growth cones that have not yet defasciculated from the $\mathrm{SNb}$ nerve.

To further examine whether RP3 axon guidance might be altered in dock mutants, we used microinjection techniques to fill RP3 neurons with dye. Using digital optical microscopy with Nomarski contrast enhancement (Chiba et al., 1993), we 

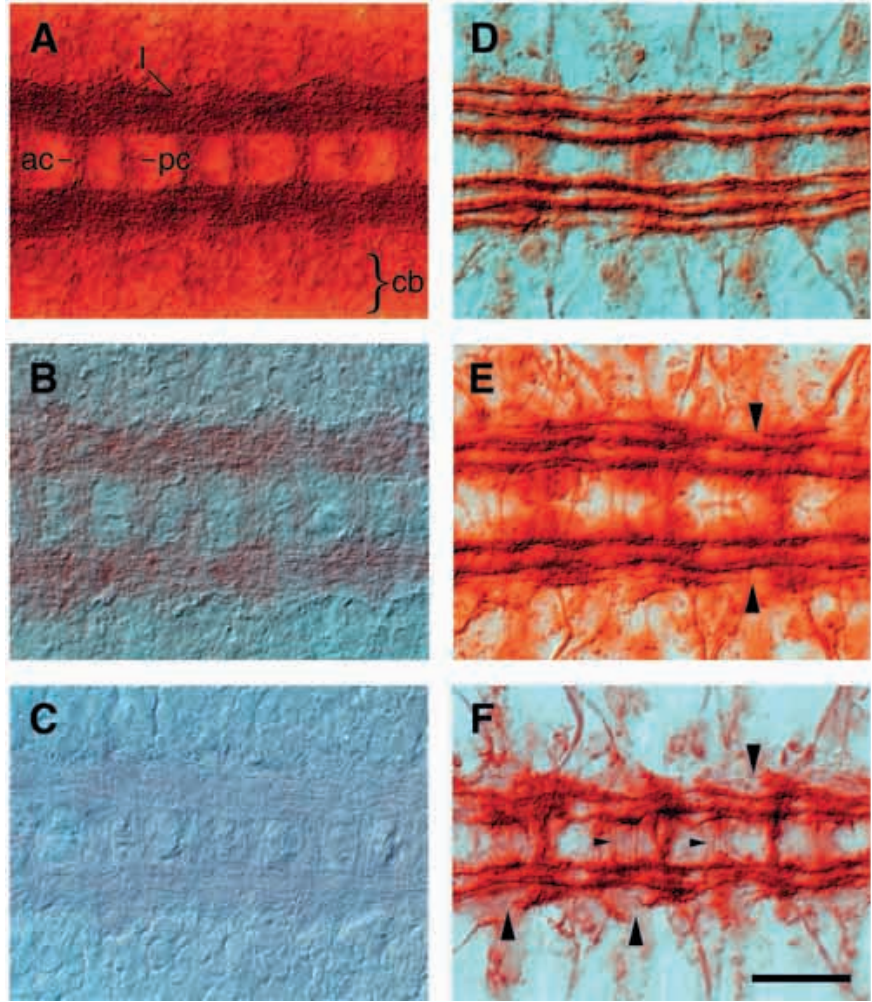

Fig. 1. Dock protein expression and dock phenotypes in the CNS. The ventral nerve cords of wild-type (A,D), zygotic loss of dock $(\mathrm{B}, \mathrm{E})$ and zygotic and maternal loss of $\operatorname{dock}(\mathrm{C}, \mathrm{F})$ late stage 16 embryos were stained with anti-Dock antiserum (A-C) or mAb 1D4 (D-F), using HRP immunohistochemistry for detection, and photographed using DIC optics. (A) Anti-Dock labels the longitudinal axon tracts (1), anterior commissures (ac), posterior commissures (ac) and cell bodies (cb), consistent with expression by most if not all CNS neurons. (B) Axonal Dock is still detectable in this $\operatorname{dock}^{P 1} /$ dock $^{P 1}$ embryo, although at much reduced levels. (C) No Dock expression is visible in embryos lacking both maternal and zygotic dock. function. (D) mAb 1D4 stains three pairs of axon bundles running longitudinally in the CNS. (E) The three bundles are present in zygotic dock mutants, although they are wavier and more varied in thickness than in wild type, and display occasional breaks in the outermost bundle (large arrowhead). This is a dock $^{P 2} /$ dock $^{3}$ embryo. (F) The bundles are very disorganized in embryos lacking both maternal and zygotic expression of Dock. The outermost bundle is discontinuous (large arrowheads) and FasII-expressing axons are seen crossing the midline (small arrowheads). Anterior is to the left. Scale bar, $20 \mu \mathrm{m}$.

cleft, indicating that all axonal guidance events leading to the arrival of the growth cone at the synaptic target had proceeded normally. However, the growth cones had not penetrated the $7 / 6$ cleft to the internally facing side, a feature that normally precedes synaptogenesis, in any of the preparations that we examined (Fig. 6B-D). These results indicate that the absence of RP3 synapses is due to a defect in terminal guidance and/or were able to identify RP3 motoneuron cell bodies in all segments examined in live, filleted dock embryos. RP3 neurons were dye-filled with Lucifer Yellow (LY) and, in every case $(n=15)$, were found to have normal morphology within the CNS (Fig. 6A). These features include a normally located cell body (Fig. 6A, arrow), a contralateral axonal projection within the anterior commissure, appropriate contralateral dendritic arborization (Fig. 6A, arrowhead) and an axonal trajectory along the ISN root to exit the CNS. In the periphery, we found that RP3 growth cones were appropriately located at the muscle fiber $7 / 6$

Fig. 2. Dock protein expression in the periphery of late stage 16 embryos. Embryos were stained with antiDock antiserum. (A) Dock is expressed on the ISN motor nerve and appears to be concentrated at the terminal arbor (T), and first branchpoint (FB). These are both synaptic sites where growth cones are paused. Dock is also expressed on sensory axons from the dorsal cluster (arrowheads). (B) Dock is highly expressed at muscle attachment sites (arrows). The multiple dendrite cell (m) also expresses Dock.

(C) PNS cell bodies including the chordotonal organs (c), dorsal cluster (d) and multiple dendrite cell (m) express Dock. (D) Dock expression in the $\mathrm{SNb}$ motor nerve is concentrated in growth cones and developing synapses along muscle clefts (arrowheads). Note that Dock is expressed on the RP3 synapse forming in the cleft between muscles 7 and 6 (red arrowhead). Muscle fibers are labeled with numbers and muscle attachment sites with arrows in all panels. Anterior is to the left, dorsal is up. Scale bar, $10 \mu \mathrm{m}$.
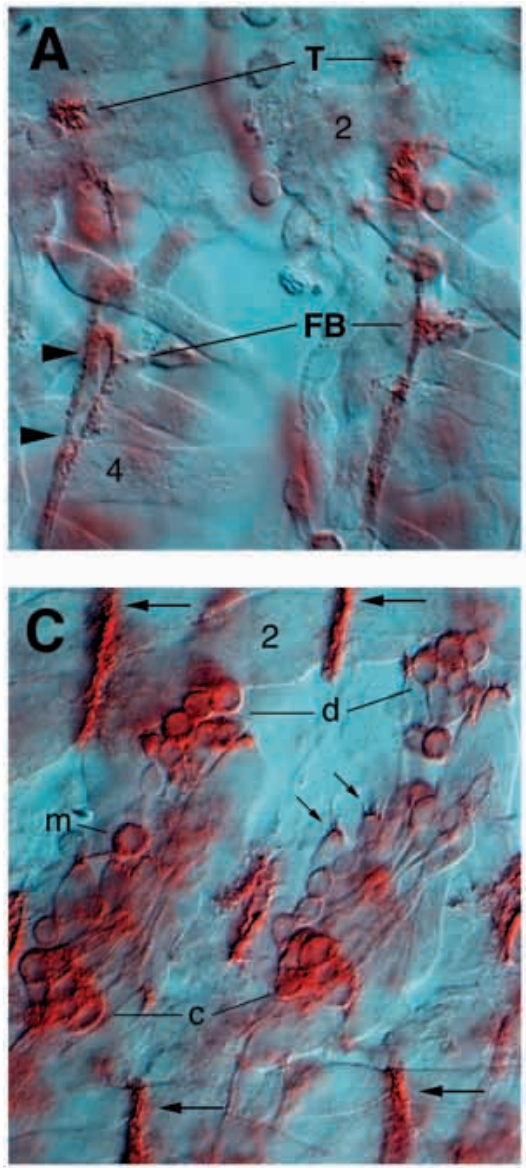

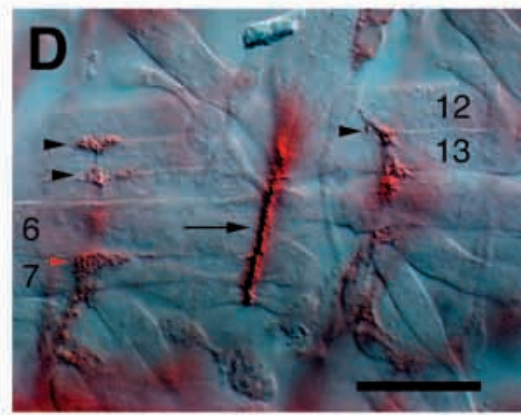



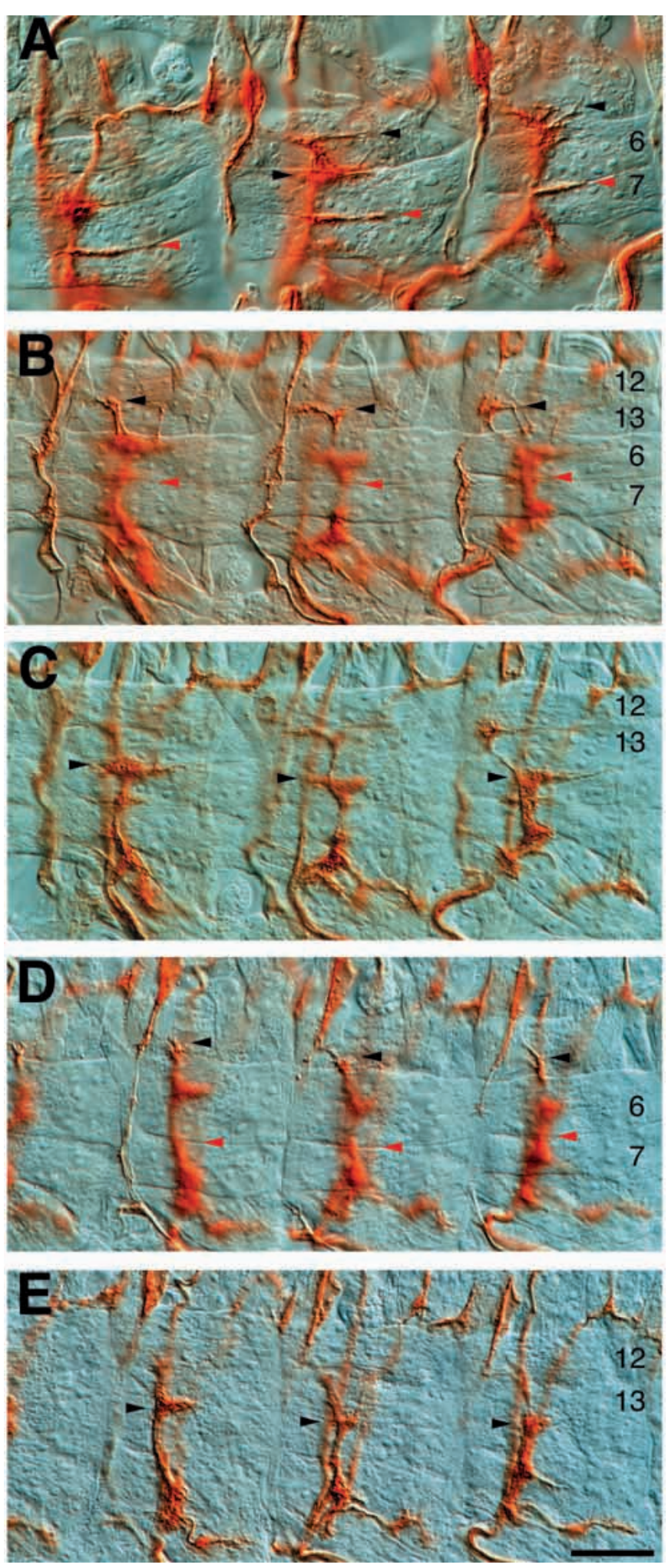

differentiation of the RP3 growth cones, rather than to alterations in their axonal outgrowth from the CNS.

We next wished to determine whether RP3 synaptogenesis was permanently blocked or merely delayed in the hemisegments of dock mutants that lack innervation of the 7/6 cleft at late stage 16. To analyze this, we first examined the neuromuscular system in dissected dock mutant third-instar
Fig. 3. Synapse formation by SNb motor axons in wild-type and mutant embryos. Three abdominal hemisegments of wild-type (A), $\operatorname{dock}^{P 2} / \operatorname{dock}^{3}(\mathrm{~B}, \mathrm{C})$ and $\mathrm{lbm}^{Y 13}(\mathrm{D}, \mathrm{E})$ late stage 16 embryos stained with MAb 1D4 are shown. (A) Wild type (Oregon R). Synapse elongation is nearly complete along the $7 / 6$ cleft (red arrowheads) in this wild-type embryo, but synapses are only beginning to appear at the 13/12 cleft (left-pointing black arrowheads). Synaptogenesis is well underway along the proximal edge of muscle 13 as well (rightpointing black arrowhead), but this structure is out of focus in this image. (B) dock, superficial focal plane. Synapse formation along the $13 / 12$ cleft (black arrowheads) is more advanced than in panel (A), but no synapses are visible at the 7/6 clefts (red arrowheads).

(C) dock, deeper focal plane (same embryos as B). Synapses forming along the proximal edge of muscle 13 are visible (black arrowheads). (D) $\mathrm{lbm}$, superficial focal plane. SNb growth cones are still exploring muscle 13 (black arrowheads) and have not yet begun synaptogenesis along the $13 / 12 \mathrm{cleft}$, indicating that this $\mathrm{lbm}$ embryo is slightly younger than the embryo in (A). The 7/6 cleft is uninnervated in all three hemisegments (red arrowheads). (E) $\mathrm{lbm}$, deeper focal plane. Synapses have begun to form along the proximal edge of muscle 13 (black arrowheads). Ventrolateral muscles are indicated by numbers. Anterior is to the left, dorsal is up. Scale bar, $8 \mu \mathrm{m}$.
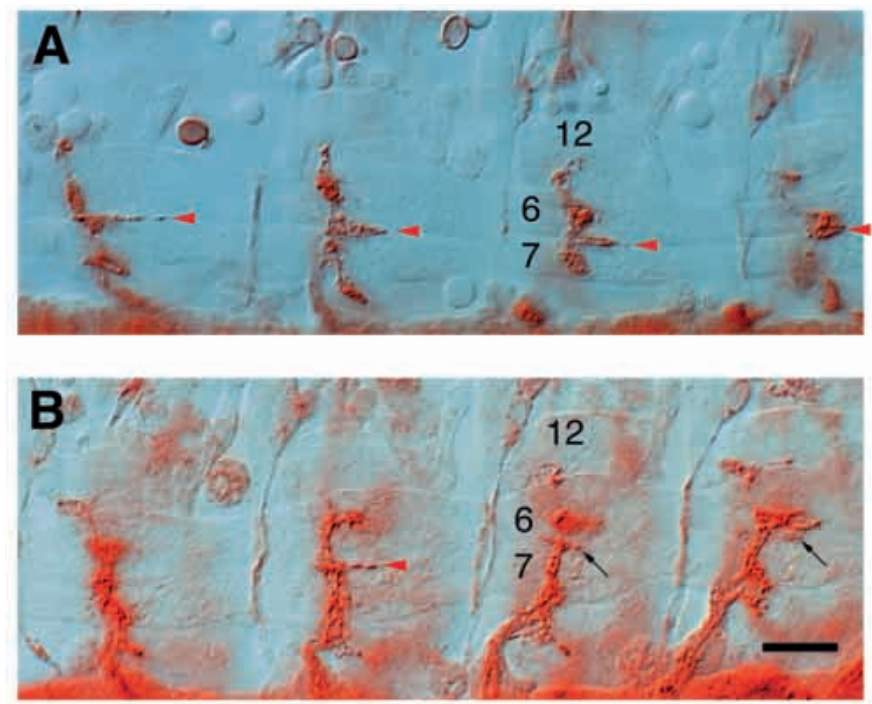

Fig. 4. Synaptotagmin staining of 7/6 cleft synapses is reduced in dock mutant embryos. Four abdominal hemisegments of late stage 16/early stage 17 embryos stained with anti-Syt are shown. (A) In a wild-type embryo, a normally sized synapse is present in all four hemisegments (red arrowheads). (B) In a $d o c k^{P 1}$ embryo, no Syt staining at the $7 / 6$ cleft is seen in the left-hand hemisegment, and very weak staining in the two right-hand hemisegments (black arrows). In the second hemisegment from the left, stronger Syt staining at the cleft is seen (red arrowhead), although the synapse is still smaller than in most wild-type hemisegments. Muscle fibers 6, 7 and 12 are labeled. Scale bar, $8 \mu \mathrm{m}$.

larvae. It has been previously shown that larval muscles that have been deprived of their normal synaptic input for several hours after hatching display ectopic innervation by other motor nerves (Chang and Keshishian, 1996). The innervation of muscles 7 and 6 by RP3 is apparently normal in dock mutant third-instar larvae, and no ectopic synapses are observed (data not shown). This indicates that RP3 can always form synapses in these mutants and that these synapses have all developed by hatching or shortly thereafter. 
Table 1. Synaptogenesis by the RP3 growth cone

\begin{tabular}{|c|c|c|c|c|c|}
\hline Genotype & $\begin{array}{l}7 / 6 \text { cleft innervation } \\
\text { score }(\% \text { reduction })^{\mathrm{a}}\end{array}$ & $n$ & $\begin{array}{l}\% \text { total RP synaptic } \\
\text { length due to RP3 }\end{array}$ & $n^{\mathrm{c}}$ & $\begin{array}{l}\% \text { of } 7 / 6 \text { clefts } \\
\text { with no synapse }\end{array}$ \\
\hline Oregon $R$ & 1.44 & 80 & $51 \%$ & 73 & $10 \pm 6 \%$ \\
\hline $\operatorname{dock}^{P 1}$ & $0.55(62 \%)$ & 87 & $13 \%$ & 71 & $45 \pm 11 \% * *$ \\
\hline $\operatorname{dock}^{P 2}$ & $1.25(13 \%)$ & 160 & $29 \%$ & 108 & $38 \pm 11 \% * *$ \\
\hline$d o c k^{3}$ & $1.06(26 \%)$ & 87 & $26 \%$ & 108 & $41 \pm 10 \% * *$ \\
\hline $\operatorname{dock}^{P 2} / \operatorname{dock}^{3}$ & $1.07(27 \%)$ & 78 & $29 \%$ & 86 & $28 \pm 10 \% *$ \\
\hline $\operatorname{dock}^{P 1}\left(\right.$ ovo $\left.^{D}\right) /$ dock $^{P 2}$ & $0.88(39 \%)$ & 96 & $22 \%$ & 67 & $44 \pm 12 \% * *$ \\
\hline $\mathrm{lbm}^{Y 13}$ & $0.47(67 \%)$ & 79 & $26 \%$ & 105 & $49 \pm 10 \% * *$ \\
\hline $\begin{array}{l}\operatorname{dock}^{P 1} \mathrm{lbm}^{Y 13} / \operatorname{dock}^{P 2} \mathrm{lbm}^{Y 13} \\
\operatorname{dock}^{P 1}\left(\text { ovo }^{D}\right) / \text { dock }^{P 2}\end{array}$ & $\begin{array}{l}0.58(60 \%) \\
\text { N.A. }\end{array}$ & 103 & $\begin{array}{l}21 \% \\
41 \%\end{array}$ & $\begin{array}{l}84 \\
60\end{array}$ & $\begin{array}{c}47 \pm 11 \% * * \\
0 \%\end{array}$ \\
\hline
\end{tabular}

(cuticulated st. 17 embryos) $)^{\mathrm{e}}$

annervation of the 7/6 cleft in hemisegments A3-A6 of late stage 16/early stage 17 embryos was quantified using the following scheme:

$0=$ no synapse or growth cone in or above the $7 / 6 \mathrm{cleft}$

$1=$ short or thin branch starting in the $7 / 6$ cleft

$2=$ a long, thick $7 / 6$ branch (such as those in Fig. 3A)

Each genotype was then normalized to Oregon R, the wild-type control. Oregon R scored only 1.44 on this scale, indicating that RP3 growth cone extension along the 7/6 cleft is not complete at this stage in wild-type embryos. We scored only segments A3-A6, rather than A2-A7, because the DC1 motoneuron sometimes innervates the 7/6 cleft in A2, and A7 synaptic development is often delayed relative to A2-A6. To increase the objectivity of our scores, embryos were scored blindly by three members of the Zinn group. Although the absolute scores varied somewhat, the rank order was identical in each case. The numbers reported in this table are derived from the blind scores of one person (C. J. D.). The percentage reduction in RP3 synapse formation relative to Oregon R is indicated in parentheses, and was calculated as follows:

(1-(score for mutant $7 / 6$ cleft innervation $/ 1.44$ [score for Oregon $\mathrm{R}])) \times 100 \%=\%$ reduction.

bThe proportion of total synaptic length elaborated by RP1, RP3, RP4 and RP5 that was due to RP3 was determined in order to ensure that the delay in $7 / 6$ innervation was selective rather than a global delay in SNb synaptogenesis. If formation of all RP synapses were delayed to the same extent in $d o c k$ and $l b m$ mutants, this number would be the same for all genotypes. The synaptic lengths formed by RP3 along the 7/6 cleft (SL3), RP1 and 4 along the proximal edge of muscle 13 (SL1\&4) and RP5 along the 13/12 cleft (SL5) were measured. Thus,

$\%$ total SNb synaptic length due to RP3=(SL3/(SL3+SL1\&4+SL5)) $\times 100 \%$

'These ' $n$ ' values refer to the number of A3-A6 hemisegments scored for " $\%$ total RP synaptic length due to RP3" (column 4). This ' $n$ ' differs from the 'n' in

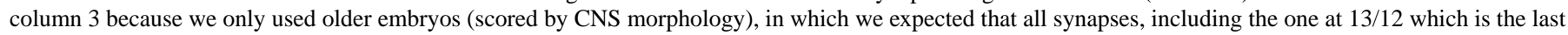
to form, would have appeared. For some genotypes, additional older embryos were dissected and scored in order to obtain larger ' $n$ 's' for column 4.

dThe percentage of hemisegments devoid of synapses in the 7/6 cleft and the $95 \%$ confidence intervals are shown for each genotype.

**Chi-square analysis indicates that mutant and control values differ significantly $(P<0.0001)$.

*Chi-square analysis indicates that mutant and control values differ significantly $(P<0.005)$.

${ }^{e}$ Cuticulated embryos were stained after dissection. Since every hemisegment examined displayed some innervation (column 5 ) and the number in column 4 of this row $(41 \%)$ is higher than for the same genotype when scored at late stage 16/early stage 17 (29\%), the RP3 synapse is clearly continuing to mature in embryos that lack both maternal and zygotic Dock.

To determine when RP3 might form synapses in the hemisegments of dock mutant embryos in which the 7/6 cleft is unninervated at stage 16, we also examined embryos that had already secreted cuticle and acquired a morphology resembling that of first-instar larvae. Cuticle secretion begins about 17 hours after egg deposition, coincident with the constriction of motor growth cone pre-varicosities into varicosities. Cuticulated stage 17 embryos are impermeable to antibodies as whole mounts, but we were able to stain them with mAb 1D4 after dissection. We found that the 7/6 cleft always contains a synapse in cuticulated stage 17 dock mutants, even those lacking both maternal and zygotic Dock (see below). The average length of this synapse relative to the total length of all RP synapses is still less than in wild-type stage 16/17 embryos, however (Table 1).

\section{dock and late bloomer cause similar defects}

The delay in RP3 synapse formation (lack of 7/6 cleft innervation at late stage 16 , but normal innervation in third instar larvae) seen in dock mutant embryos is identical to the phenotype of late bloomer $(\mathrm{lbm})$ mutants (Kopczynski et al., 1996). lbm encodes a member of the tetraspanin family that is expressed on motoneurons. Fig. 3D shows three hemisegments in an $\mathrm{lbm}$ mutant that lack synapses at the $7 / 6$ cleft (red arrowheads). $d o c k^{P 1} / d o c k^{P 2}$ and $l b m^{Y 13}$ late stage 16 embryos display the 7/6 innervation defect at similar penetrances $(68 \%$ and $67 \%$, respectively). $49 \%$ of hemisegments in $\mathrm{lbm}^{Y 13}$ embryos completely lack synapses at the 6/7 cleft (Table 1). Like dock mutants, $l \mathrm{lbm}$ embryos also have low-penetrance body wall muscle defects.

The delay in synapse formation seen in dock and $\mathrm{lbm}$ mutants might indicate that Dock and Lbm function in partially redundant biochemical pathways necessary for RP3 synaptogenesis. If so, embryos lacking both Lbm and Dock should display more penetrant and/or more severe defects than either single mutant. To test this hypothesis, we examined the neuromuscular system in dock lbm double mutants. We found that neither the delay of RP3 synapse formation nor the occasional muscle and $\mathrm{SNb}$ guidance defects were more penetrant in double mutants than in $l b m$ or dock single mutants (Table 1). Fig. 5A shows a double mutant stained with antiFasIII. Guidance of RP axons is normal in these mutants.

Like dock larvae, dock $\mathrm{lbm}$ third instar larvae display no ectopic innervation of muscles 7 and 6 (data not shown), indicating that the RP3 defects observed in double mutant embryos are also due to delayed rather than blocked synapse 
formation. Finally, no obvious additional defects were observed in other axon pathways. These observations indicate that Dock and Lbm are not essential components of separate, partially redundant pathways required for RP3 synaptogenesis.

\section{Maternal loss of dock expression enhances axon defects}

In the course of selecting homozygous dock mutant embryos for dissection, we noticed that none of the progeny of mothers heterozygous for dock alleles completely lacked Dock protein expression. Fig. 1B shows Dock protein in the CNS of a $\operatorname{dock}^{P 1}$ embryo ( $\operatorname{dock}^{P I}$ is the most severe dock allele). Dock is clearly detectable in CNS axons and at muscle attachment sites (not shown) in the mutant embryo, although the level of expression is much lower than in wild type (compare Fig. 1A to B). One explanation for the presence of Dock protein in embryos homozygous for dock mutations is that they receive a maternal contribution of Dock mRNA and/or protein that persists late in embryogenesis. Persistent maternal Dock could account for the incomplete penetrance and/or the mild nature of the RP3 defect observed in dock embryos.

To determine the origin of the anti-Dock reactivity and to discover the complete loss-of-function dock phenotype, we generated females heterozygous for dock mutations that had homozygous dock mutant ovaries using the FLP/ovo ${ }^{D}$ system (Chou and Perrimon, 1996). Such females produce oocytes devoid of Dock message and protein which, when fertilized with dock mutant sperm, develop into complete-loss-offunction dock mutant embryos. The CNS in such embryos is not stained by anti-Dock (Fig. 1C), indicating that the residual staining in zygotic loss-of-function dock mutants is indeed due to maternally derived Dock rather than to a cross-reacting epitope.

When we examined mutant embryos lacking both the maternal and zygotic contributions for dock, we noticed marked CNS axon defects. The outermost FasII-positive axon bundles are severely discontinuous (large arrowheads in Fig. 1F). In addition, it appears that some FasII-positive axons cross the midline that do not normally do so (small arrowheads). Although the pattern of motor nerves is still fairly normal, complete dock null embryos do display increased levels of $\mathrm{SNb}$ abnormalities, some of which might result from guidance errors within the ventral cord. For example, a number of embryos display segments in which the $\mathrm{SNb}$ on one side is abnormally thick while the contralateral $\mathrm{SNb}$ is abnormally thin (data not shown). This phenotype could be explained if some $\mathrm{SNb}$ axons failed to cross the midline and contributed to the ipsilateral $\mathrm{SNb}$ instead. Maternal loss of Dock also

Table 2. Synaptotagmin expression at $\mathbf{7 / 6}$ cleft synapses

\begin{tabular}{lcccc}
\hline Genotype & No synapse & Weak synapse & Normal synapse & $n$ \\
\hline dock $^{P I}$ & $37 \pm 13 \%$ ** & $37 \pm 13 \%$ & $26 \pm 12 \%$ ** & 54 \\
dock $^{P 1} /$ Balancer & $7 \pm 6 \%$ & $29 \pm 11 \%$ & $64 \pm 11 \%$ & 70
\end{tabular}

Embryos were dissected alive, fixed and immunostained for Syt. The fillets were then reprocessed to visualize Dock protein, in order to score genotype. "Weak synapse" corresponds to class 1 in Table 1 (see note 'a'). The mean and the $95 \%$ confidence intervals are shown for each phenotype.

**Chi-square analysis indicates that mutant and control value differs significantly $(P<0.0001)$. enhances dock-induced lethality. Zygotic dock mutants usually survive until the pupal stage, while embryos lacking both maternal and zygotic Dock fail to hatch.

By contrast, the inhibition of RP3 synapse formation is not worsened in late stage 16/early stage 17 embryos lacking both maternal and zygotic Dock. The innervation of the $7 / 6 \mathrm{cleft}$ in $\operatorname{dock}^{P 1}\left(\right.$ ovo $\left.^{D}\right) /$ dock $^{P 2}$ embryos is reduced by $39 \%$ relative to wild-type embryos of the same stage (Table 1), and $44 \%$ of $7 / 6$ clefts completely lack synapses. This penetrance is actually lower than that displayed by zygotic $\operatorname{dock}^{P 1} / \operatorname{dock}^{P 2}$ embryos, indicating that the presence of maternally derived Dock is not responsible for $7 / 6$ cleft innervation in zygotic dock mutants. The 7/6 cleft also shows increased innervation in dissected cuticulated stage $17 \operatorname{dock}^{P 1}\left(\right.$ ovo $\left.^{D}\right) / \operatorname{dock}^{P 2}$ embryos relative to late-stage 16 embryos of the same genotype (Table 1), so we conclude that RP3 synaptogenesis is only delayed rather than blocked even when both maternal and zygotic Dock are absent. Note that, because maternalplus-zygotic dock embryos fail to hatch, we could not examine third-instar larvae to determine if all RP3 neurons eventually innervated the $7 / 6$ cleft.

The other tissues that normally express Dock likewise appear normal in complete dock null embryos. The body wall musculature appears intact despite the loss of Dock at muscle attachment sites. The PNS also appears largely normal, although there is some variability in chordotonal organ neuron numbers. $8 \%$ of hemisegments in complete null embryos had four chordotonal neurons instead of five, and $6 \%$ had six chordotonal neurons $(n=178)$.

\section{DISCUSSION}

The Dock SH2-SH3 domain adapter protein is prominently expressed in the embryonic CNS (Fig. 1). In motor axons, Dock is concentrated in growth cones (Fig. 2). Embryos unable to synthesize Dock display a specific impairment in formation of a single neuromuscular synapse, that of the RP3 motoneuron at the cleft between muscles 7 and 6 (Figs 3, 4; Tables 1, 2). The absence of the RP3 synapse is corrected later in development, so that $7 / 6$ cleft innervation in dock mutant larvae appears indistinguishable from wild type.

We do not observe alterations in RP axon guidance in dock mutants (Figs 5, 6). The mutants display a slight disorganization of FasII-expressing longitudinal axon bundles in the CNS (Fig. 1), and these longitudinal axon defects are strikingly enhanced in embryos lacking both zygotic and maternal Dock. In these complete-loss-of-function dock embryos, the outermost FasII-positive longitudinal axon bundles almost never form and FasII-positive axons abnormally cross the midline (Fig. 1).

The enhanced CNS defects in embryos lacking both maternal and zygotic sources of Dock suggests that residual maternally derived Dock protein persists in dock mutant embryos derived from heterozygous mothers. Indeed, CNS axons in stage 16 zygotic dock mutant embryos display clearly visible Dock expression, whereas no expression is detectable in maternal-plus-zygotic dock embryos (Fig. 1). It is often assumed that maternally contributed mRNAs and proteins are diluted or degraded prior to axonogenesis, but this is clearly not the case for Dock. 

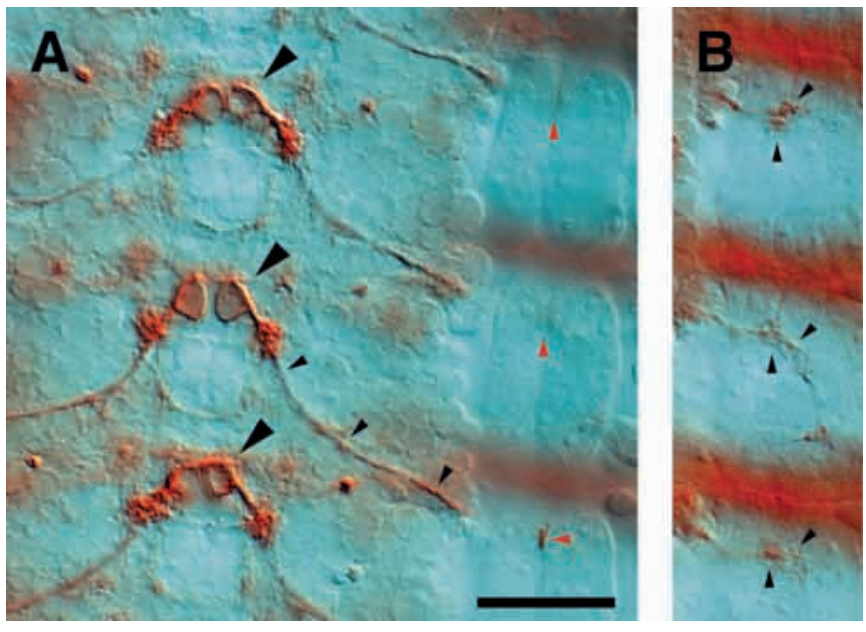

Fig. 5. RP axon guidance is normal in dock lbm double mutants. Three segments of a late stage 16 embryo stained with anti-FasIII are shown. (A) Two clusters of RP neuron cell bodies present in each segment express FasIII normally (large arrowheads) and extend axons along normal trajectories (small black arrowheads). Synapse formation has begun in the 7/6 cleft in one hemisegment (horizontal red arrowhead) but not at the other two (upward red arrowheads). (B) FasIII-expressing RP axons exploring beyond the 7/6 cleft (diagonal arrowheads) are visible in this deeper focal plane. Note the accumulation of FasIII-expressing material directly below the 7/6 cleft (upward arrowheads). These are probably paused RP3 growth cones. Anterior is up. Scale bar, $20 \mu \mathrm{m}$.

\section{RP3 synaptogenesis is sensitive to genetic perturbation}

The transient, selective delay in RP3 synapse formation is shared by embryos lacking either Dock or the tetraspanin Lbm (Kopczynski et al., 1996; Fig. 3). This shared phenotype could be explained if the two proteins participate in redundant pathways mediating RP3 synapse maturation. Since dock lbm double mutants do not have more severe phenotypes than either single mutant (Table 1; Fig. 5), however, our results do not support such a model. Instead, Lbm and Dock might participate in a common pathway that contributes to, but is not essential for, RP3 synapse formation. The mild defects arising from absence of both Dock and Lbm indicates that other pathways are sufficient to allow RP3 synaptogenesis. Such signaling pathways must also mediate development of the other neuromuscular synapses. All of the other motor growth cones, including those of the ISN, $\mathrm{SNa}, \mathrm{SNc}, \mathrm{SNd}$ and $\mathrm{SNb}$ motoneurons, express both Lbm and Dock but still form normal presynaptic terminals in $\mathrm{dock}, \mathrm{lbm}$ and double mutants.

It is interesting that loss-of-function mutations in both the dock and $\mathrm{lbm}$ genes selectively delay the formation of the same neuromuscular synapse. Netrin $B$ loss-of-function mutations also impair formation of the RP3 synapse (Winberg et al., 1998). In contrast, no mutations selectively affecting formation of any of the other individual neuromuscular junctions have been discovered. One explanation for this is that the formation of the RP3 synapse on muscles 7 and 6 may be developmentally 'difficult'. The RP3 growth cone must push between two tightly apposed muscle fibers before reaching its synaptic site, whereas most other motor growth cones simply grow over muscle surfaces until they reach their synaptic target
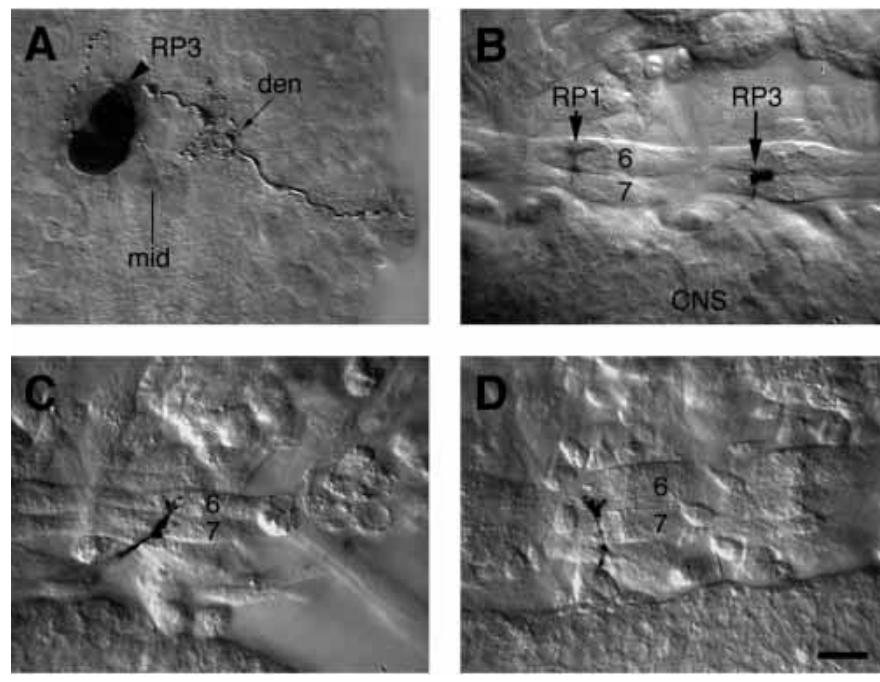

Fig. 6. Intracellular dye fills of RP motoneurons in dock mutants reveal normal axonal trajectories in late stage 16 embryos. RP motoneurons in dock mutants were filled with LY. The fill in A was processed for immunohistochemistry with anti-LY antibody. The fills in B-D are displayed as negative images of LY fluorescence superimposed over DIC images of the musculature, in living unfixed embryos. (A) RP3 dye fill showing a typical contralateral projection of the neurite across the ventral midline (mid), with a normal dendritic arborization (den) in the CNS. The second cell body shown is an adjacent dye-filled glial cell. (B) Double dye fill of motor neurons RP1 and RP3 in adjacent abdominal segments. The ending of RP1 on muscle fiber 13 (partially covered by muscle fiber 6) and of RP3 on the muscle fiber 7/6 cleft are indicated. (C,D) Examples of RP3 dye fills showing the growth cones located on the external side of the muscle fiber $7 / 6$ cleft. Anterior is to the top (A), or to the left (B-F). Scale bar, $10 \mu \mathrm{m}$ in A; $6 \mu \mathrm{m}$ in B-D.

sites. Consistent with this hypothesis, genetic perturbation studies reveal that RP3 synaptogenesis is sensitive to the strength of adhesion between muscle fibers 7 and 6 . Overexpression of the homophilic adhesion molecules Connectin and FasIII on these muscles inhibits formation of the RP3 synapse (Chiba et al., 1995; Nose et al., 1994) and, in such mutant animals, muscle fibers 7 and 6 are more tightly apposed than in wild type (Kose et al., 1997; Raghavan and White, 1997).

\section{Potential signaling functions of Dock and Lbm}

Dock and its mammalian homolog Nck, which can rescue the $\mathrm{R}$ axon defects resulting from loss of Dock (Rao and Zipursky, 1998), have been implicated in several different signaling pathways. The SH2 domains of Dock and Nck can bind to activated receptor tyrosine kinases (Stein et al., 1998). Nck interacts with proteins involved in Rho-family GTPase signaling pathways (Quilliam et al., 1996), which regulate the structure of the growth cone's cytoskeleton.

Nck also associates with activated focal adhesion kinase (FAK; Schlaepfer et al., 1997), which is an important effector for integrins. The interaction between FAK and Nck is interesting in light of the genetic evidence suggesting that Dock and the tetraspanin Lbm participate in the same processes during RP3 synaptogenesis. Tetraspanins associate with integrins, and this interaction can increase integrin signaling 
(Banerjee et al., 1997; Guan, 1997). These observations are consistent with a model in which Lbm expressed on the RP3 growth cone might facilitate integrin-mediated activation of FAK and recruitment of Dock to the FAK signaling complex.

The axon defects associated with loss of Dock in embryos and larvae suggest that a major function of the signaling pathway(s) in which Dock participates is to link tyrosine kinase activation to cytoskeletal changes in the growth cone. In dock mutant larvae, axons from R1-6 often fail to terminate in the lamina, instead remaining fasciculated with $\mathrm{R} 8$ axons and projecting into the deeper medulla neuropil (Garrity et al., 1996). R cell growth cones display morphological defects and are impaired in topographic map formation. Similarly, we show here that RP3 synaptogenesis is delayed at a point of radical change in growth cone behavior. Prior to reaching the cleft between muscle 7 and 6, the RP3 growth cone grows in conjunction with other axons. It must then defasciculate from these axons, make a sharp turn and extend along the 7/6 cleft. Subsequent maturation of the growth cone into a synapse involves extensive cytoplasmic rearrangements, including the formation of focal attachments to the muscle fibers. Involvement of Dock in any of these processes could explain the delay in RP3 synaptogenesis observed in mutant embryos.

We thank Jim Clemens, Jack Dixon and Mark Perin for antibodies, the Bloomington Stock Center and Corey Goodman's group for fly strains, and Steven Hanks and Richard Kim for helpful advice. This work was supported by NIH RO1 grant NS28182 to K. Z., by startup funds from Vanderbilt University Medical School to C. J. D. and by NIH RO1 grant NS31651 to H. K.; P. A. G. was supported by postdoctoral fellowships from the Bank of America-Giannini Foundation and the Leukemia Society of America; S. L. Z. is an Investigator of the Howard Hughes Medical Institute.

\section{REFERENCES}

Banerjee, S. A., Hadjiargyrou, M. and Patterson, P. H. (1997). An antibody to the tetraspan membrane protein CD9 promotes neurite formation in a partially integrin-dependent manner. J. Neurosci. 17, 2756-2765.

Broadie, K. S. and Bate, M. (1993). Development of the embryonic neuromuscular synapse of Drosophila melanogaster. J. Neurosci. 13, 144166.

Callahan, C. A., Muralidhar, M. G., Lundgren, S. E., Scully, A. L. and Thomas, J. B. (1995). Control of neuronal pathway selection by a Drosophila receptor protein-tyrosine kinase family member. Nature 376, 171-174.

Chang, T. N. and Keshishian, H. (1996). Laser ablation of Drosophila embryonic motoneurons causes ectopic innervation of target muscle fibers. J. Neurosci. 16, 5715-5726.

Chiba, A., Hing, H., Cash, S. and Keshishian, H. (1993). Growth cone choices of Drosophila motor neurons in response to muscle fiber mismatch. J. Neurosci. 13, 714-732

Chiba, A., Snow, P., Keshishian, H. and Hotta, Y. (1995). Fasciclin III as a synaptic target recognition molecule in Drosophila. Nature 374, 166-168.

Chou, T. B. and Perrimon, N. (1996). The autosomal FLP-DFS technique for generating germline mosaics in Drosophila melanogaster. Genetics 144, 1673-1679.

Clemens, J. C., Ursuliak, Z., Clemens, K. K., Price, J. V. and Dixon, J. E. (1996). A Drosophila protein-tyrosine phosphatase associates with an adapter protein required for axonal guidance. J. Biol. Chem. 271, 1700217005.

Desai, C. J., Gindhart, J. G., Goldstein, L. S. and Zinn, K. (1996). Receptor tyrosine phosphatases are required for motor axon guidance in the Drosophila embryo. Cell 84, 599-699.

Desai, C. J., Krueger, N. X., Saito, H. and Zinn, K. (1997). Competition and cooperation among receptor tyrosine phosphatases control motoneuron growth cone guidance in Drosophila. Development 124, 1941-1952.

Garrity, P. A., Rao, Y., Salecker, I., McGlade, J., Pawson, T. and Zipursky, S. L. (1996). Drosophila photoreceptor axon guidance and targeting requires the dreadlocks SH2/SH3 adapter protein. Cell 85, 639-650.

Guan, J.-L. (1997). Role of focal adhesion kinase in integrin signaling. Int. J. Biochem. Cell Biol. 29, 1085-1096.

Holland, S. J., Gale, N. W., Mbamalu, G., Yancopoulos, G. D., Henkemeyer, M. and Pawson, T. (1996). Bidirectional signalling through the EPH-family receptor Nuk and its transmembrane ligands. Nature $\mathbf{3 8 3}$, $722-725$.

Keshishian, H., Broadie, K., Chiba, A. and Bate, M. (1996). The Drosophila neuromuscular junction: a model system for studying synaptic development and function. Ann. Rev. Neurosci. 19, 545-575.

Kopczynski, C. C., Davis, G. W. and Goodman, C. S. (1996). A neural tetraspanin, encoded by late bloomer, that facilitates synapse formation. Science 271, 1867-1870.

Kose, H., Rose, D., Zhu, X. and Chiba, A. (1997). Homophilic synaptic target recognition mediated by immunoglobulin-like cell adhesion molecule Fasciclin III. Development 124, 4143-4152.

Krueger, N. X., Van Vactor, D., Wan, H. I., Gelbart, W. M., Goodman, C. S. and Saito, H. (1996). The transmembrane tyrosine phosphatase DLAR controls motor axon guidance in Drosophila. Cell 84, 611-622.

Landgraf, M., Bossing, T., Technau, G. M. and Bate, M. (1997). The origin, location, and projections of the embryonic abdominal motorneurons of Drosophila. J. Neurosci. 17, 9642-9655.

Nose, A., Takeichi, M. and Goodman, C. S. (1994). Ectopic expression of Connectin reveals a repulsive function during growth cone guidance and synapse formation. Neuron 13, 525-539.

Orioli, D., Henkemeyer, M., Lemke, G., Klein, R. and Pawson, T. (1996). Sek4 and Nuk receptors cooperate in guidance of commissural axons and in palate formation. EMBO J. 15, 6035-6049.

Park, S., Frisen, J. and Barbacid, M. (1997). Aberrant axonal projections in mice lacking EphA8 (Eek) tyrosine protein kinase receptors. EMBO J. 16, 3106-3114.

Patel, N. H., Snow, P. M. and Goodman, C. S. (1987). Characterization and cloning of Fasciclin III: a glycoprotein expressed on a subset of neurons and axon pathways in Drosophila. Cell 48, 975-988.

Perin, M.S., Johnston, P.A., Ozcelik, T., Jahn, R., Francke, U. and Sudhof, T.C. (1991). Structural and functional conservation of Synaptotagmin (P65) in Drosophila and humans. J. Biol. Chem. 266, 615-622.

Quilliam, L. A., Lambert, Q. T., Mickelson-Young, L. A., Westwick, J. K., Sparks, A. B., Kay, B. K., Jenkins, N. A., Gilbert, D. J., Copeland, N. G. and Der, C. J. (1996). Isolation of a NCK-associated kinase, PRK2, an SH3-binding protein and potential effector of Rho protein signaling. J. Biol. Chem. 271, 28772-28776.

Raghavan, S. and White, R. A. (1997). Connectin mediates adhesion in Drosophila. Neuron 18, 873-880.

Rao, Y. and Zipursky, S. L. (1998). Domain requirements for the Dock adapter protein in growth-cone signaling. PNAS, 2077-2082.

Schlaepfer, D. D., Broome, M. A. and Hunter, T. (1997). Fibronectinstimulated signaling from a focal adhesion kinase-c-Src complex: involvement of the Grb2, p130cas, and Nck adaptor proteins. Mol. Cell. Biol. 17, 1702-1713.

Stein, E., Huynh-Do, U., Lane, A. A., Cerretti, D. P. and Daniel, T. O. (1998). Nck recruitment to Eph receptor, EphB1/ELK, couples ligand activation to c-Jun kinase. J. Biol. Chem. 273, 1303-1308.

Van Vactor, D., Sink, H., Fambrough, D., Tsoo, R. and Goodman, C. S. (1993). Genes that control neuromuscular specificity in Drosophila. Cell 73, 1137-1153.

Winberg, M. L., Mitchell, K. J. and Goodman, C. S. (1998). Genetic analysis of the mechanisms controlling target selection: complementary and combinatorial functions of netrins, semaphorins, and IgCAMs. Cell 93, 581591

Yoshinara, M., Rheuben, M. B. and Kidokoro, Y. (1997). Transition from growth cone to functional motor nerve terminal in Drosophila embryos. $J$. Neurosci. 17, 8408-8426. 